\title{
Logical Consequence Inside Out
}

\author{
Denis Bonnay ${ }^{1}$ and Dag Westerståhl ${ }^{2}$ \\ 1 University Paris Ouest, denis.bonnay@ens.fr \\ ${ }^{2}$ University of Gothenburg, dag.westerstahl@phil.gu.se
}

Tarski's definition of logical consequence for an interpreted language rests on the distinction between extra-logical symbols, whose interpretation is allowed to vary across models, and logical symbols, aka logical constants, whose interpretation remains fixed. In this perspective, logicality come first, and consequence is a byproduct of the division between logical and extra-logical symbols. Our aim here is to lay the basis for a shift in perspective: let consequence come first, so that the demarcation of a set of constants can be viewed as the by-product of the analysis of a relation of logical consequence. The idea for extracting logical constants from a consequence relation is the following: they are the symbols which are essential to the validity of at least one inference, in the sense that replacing them or varying their interpretation would destroy the validity of that inference. Conversely, definitions of logical consequence can be construed as providing us with mappings from sets of symbols (the ones selected as logical constants) to consequence relations. ${ }^{3}$ Extraction of constants is expected to be an 'inverse' to generation of consequence relations.

In Sections 1 and 2, we introduce a general substitutional framework for the abstract study of consequence relations. Extraction of constants is presented in Section 3. It is shown that extraction thus defined does not straightforwardly provide an inverse to generation of consequence relations. To circumvent this limitation, we consider in Section 4 'richness' properties of languages that make things better. In Section 5, we prove that extraction and generation constitute a Galois connection, considering families of expansions of a language, instead of a single language at a time. This gives us the correspondence for non-substitutional Tarskian consequence as a limit case.

\section{Preliminaries}

\subsection{Languages}

In the Bolzano setting, languages are interpreted; in particular every sentence is either true or false. We shall need very few assumptions about what sentences look like or how they are structured. For definiteness, a language $L$ has a set Sent $_{L}$ of sentences, which are finite strings of signs, some of which, called symbols, belong to a set $S y m b_{L}$. Let $u, v, u^{\prime}, \ldots$ vary over $S y m b b_{L}, \varphi, \psi, \ldots$ over $S e n t_{L}$, and $\Gamma, \Delta, \ldots$ over finite subsets sets of $\operatorname{Sent}_{L} . V_{\varphi}$ is the set of symbols occurring in $\varphi$; likewise $V_{\Gamma}=\cup\left\{V_{\varphi}: \varphi \in \Gamma\right\}$. $\operatorname{Tr}_{L} \subseteq \operatorname{Sent}_{L}$ is the set of true sentences in $L$.

\footnotetext{
3 This "ternary" view of logical consequence is advocated in particular by van Benthem
} [2], who traces it back to Bolzano. 


\subsection{Replacement}

We need a notion of 'appropriate' replacement of symbols by other symbols. To this end, think of $S y m b_{L}$ as partitioned into a set of categories. Then, a replacement is a partial function $\rho$ from $\operatorname{Symb}_{L}$ to $\operatorname{Symb}_{L}$ such that for $u \in$ $\operatorname{dom}(\rho), u$ and $\rho(u)$ belong to the same category. $\varphi[\rho]$ is the result of replacing each occurrence of $u$ in $\varphi$ by $\rho(u)$. It is convenient to assume that $V_{\varphi} \subseteq \operatorname{dom}(\rho)$ - in words, $\rho$ is a replacement for $\varphi$ - so that $\rho$ is the identity on symbols that don't get replaced. We may then assume that the following conditions hold: ${ }^{4}$

(1) a. If $\rho$ is a replacement for $\varphi, \varphi[\rho] \in$ Sent and $V_{\varphi[\rho]}=\operatorname{range}\left(\rho\left\lceil V_{\varphi}\right)\right.$

b. $\varphi\left[i d_{V_{\varphi}}\right]=\varphi$

c. If $\rho, \sigma$ agree on $V_{\varphi}$, then $\varphi[\rho]=\varphi[\sigma]$.

d. $\varphi[\rho][\sigma]=\varphi[\sigma \rho]$, when $\sigma$ is a replacement for $\varphi[\rho]$

\subsection{Consequence relations}

Definition 1. A relation $R \subseteq \wp\left(\right.$ Sent $\left._{L}\right) \times$ Sent $_{L}$ is

1. reflexive iff for all $\varphi \in \operatorname{Sent}_{L}, \varphi R \varphi ;{ }^{5}$

2. transitive iff whenever $\Delta R \varphi$ and $\Gamma R \psi$ for all $\psi \in \Delta$, we have $\Gamma R \varphi$;

3. monotone iff $\Delta R \varphi$ and $\Delta \subseteq \Gamma$ implies $\Gamma R \varphi$;

4. truth-preserving iff whenever $\Gamma R \varphi$ and (every sentence in) $\Gamma$ is true, $\varphi$ is also true.

Definition 2. A consequence relation in $L$ is a reflexive, transitive, monotone, and truth-preserving relation between finite sets of $L$-sentences and $L$-sentences. ${ }^{6}$

Let $\Rightarrow, \Rightarrow^{\prime}, \ldots$ vary over the set $C O N S_{L}$ of consequence relations in $L$. Define:

(2) a. $\quad \Gamma \Rightarrow^{\max } \varphi$ iff it is not the case that $\Gamma$ is true and $\varphi$ is false.

b. $\Gamma \Rightarrow^{\min } \varphi$ iff $\varphi \in \Gamma$.

$\Rightarrow^{\max }$ is essentially material implication.

Proposition 1. $\Rightarrow^{\max }, \Rightarrow^{\min } \in C O N S_{L}$, and $\left(C O N S_{L}, \subseteq\right)$ is partial order with $\Rightarrow^{\text {min }}$ as its smallest and $\Rightarrow^{\text {max }}$ as its largest element.

\section{Bolzano Consequence}

\subsection{Definition of $\Rightarrow$}

The following definition should be familiar, except that (a) it is substitutional rather than model-theoretic; (b) it allows any set of symbols to be treated as logical.

\footnotetext{
${ }^{4}$ Essentially the conditions in Peter Aczel's notion of a replacement system from [1].

${ }^{5}$ Writing $\psi R \varphi$ instead of $\{\psi\} R \varphi$.

${ }^{6}$ The notion of a consequence relation could be varied in various ways, but in this paper we stick to Definition 2.
} 
Definition 3. For any $X \subseteq S y m b_{L}$, define the relation $\Rightarrow_{X}$ by

$\Gamma \Rightarrow_{X} \varphi$ iff for every replacement $\rho$ (for $\Gamma$ and $\varphi$ ) which is the identity on $X$, if $\Gamma[\rho]$ is true, so is $\varphi[\rho]$.

A relation of the form $\Rightarrow_{X}$ is called a Bolzano consequence (relation); BCONS $S_{L}$ is the set of Bolzano consequences. ${ }^{7}$

Proposition 2. (a) $B C O N S_{L} \subseteq C O N S_{L}$

(b) In addition, Bolzano consequence is base monotone:

$X \subseteq Y$ implies $\Rightarrow_{X} \subseteq \Rightarrow_{Y}$

(c) $\left(B C O N S_{L}, \subseteq\right)$ is a partial order with $\Rightarrow_{\emptyset}$ as its smallest and $\Rightarrow_{\text {Symb }}$ as its largest element.

$\left(B C O N S_{L}, \subseteq\right)$ is a sub-order of $\left(C O N S_{L}, \subseteq\right)$, and $\Rightarrow^{\max }=\Rightarrow_{S y m b}$, although usually $\Rightarrow^{\text {min }} \subsetneq \Rightarrow \emptyset$. The following two lemmas are trivial but fundamental:

Lemma 1. (Replacement Lemma) If $\Gamma \Rightarrow_{X} \varphi$ and $\rho$ doesn't move any symbols in $X$, then $\Gamma[\rho] \Rightarrow_{X} \varphi[\rho]$.

Lemma 2. (Occurrence Lemma) $\Gamma \Rightarrow_{X} \varphi$ if and only if $\Gamma \Rightarrow_{X \cap V_{\Gamma \cup\{\varphi\}}} \varphi$.

\section{$2.2 \quad$ Examples}

Propositional logic A standard language of propositional logic has symbols in a set $X_{0}$ of connectives, say, $X_{0}=\{\neg, \wedge, \vee\}$, and an infinite supply $p_{0}, p_{1}, \ldots$ of propositional letters. The usual definition of logical consequence, $\models_{P L}$, is modeltheoretic, but we can 'simulate' it in the present substitutional setting, where $p_{0}, p_{1}, \ldots$ are sentences with fixed truth values. Assuming that the sequence of truth values of $p_{0}, p_{1}, \ldots$ is not eventually constant, one easily verifies that

$$
\Gamma \models_{P L} \varphi \text { iff } \Gamma \Rightarrow_{X_{0}} \varphi
$$

First-order logic For first-order logic the symbols are, say, $X_{1}=X_{0} \cup\{\exists, \forall,=\}$, and a supply of predicate symbols and individual constants. Now there is a difference between model-theoretic and substitutional definitions: in general we have

(4) $\quad \models_{F O} \subsetneq \models_{F O_{\text {subst }}} \subsetneq \Rightarrow_{X_{1}}$

where $\models_{F O_{\text {subst }}}$, i.e. the consequence relation you get with a standard substitutional interpretation of the quantifiers, as in [3]. So $\models_{F O}$ is a consequence relation, but not a Bolzano consequence.

\footnotetext{
${ }^{7}$ For readability, we use ' $\Rightarrow_{X}$ in two ways: as a relation symbol, which enables us to write things like $\Gamma \Rightarrow_{X} \varphi$, and as the value of the function $\Rightarrow_{-}: S y m b_{L} \longrightarrow B C O N S_{L}$ for the argument $X$.
} 


\subsection{Minimal sets of symbols}

Different sets may generate the same Bolzano consequence, so one expect sets that are minimal in this respect to be particularly well behaved.

Definition 4. $X$ is minimal iff for all $u \in X, \Rightarrow_{X} \neq \Rightarrow_{X-\{u\}}$.

Proposition 3. $X$ is minimal iff no proper subset of $X$ generates the same consequence relation.

The next result shows that it is sufficient to look at consequence relations generated by minimal sets.

Proposition 4. Every $X \subseteq S y m b_{L}$ has a subset which is minimal among those generating $\Rightarrow_{X}$.

A stricter notion of minimality is the following:

Definition 5. $X$ is strongly minimal iff for all $u \in X$ there are $\Gamma, \varphi$, and $u^{\prime}$ such that $\Gamma \Rightarrow_{X} \varphi, \Gamma\left[u / u^{\prime}\right]$ is true, but $\varphi\left[u / u^{\prime}\right]$ is false. ${ }^{8}$

The following is practically immediate.

(5) If $X$ is strongly minimal, it is minimal.

Let $M I N_{L}\left(S M I N_{L}\right)$ be the set of (strongly) minimal subsets of $S y m b_{L}$. Strong minimality says that $\Rightarrow_{X} \subseteq \Rightarrow_{X-\{u\}}$ fails in a particular way: a counterexample exists which involves replacing only $u$. One can show that, unless extra assumptions are made about the language (Section 4 below), not all Bolzano consequences are of the form $\Rightarrow_{X}$ for strongly minimal $X$. But those of this form are particularly well behaved:

Proposition 5. If $X$ is strongly minimal then, for all $Y \subseteq S_{y m b}, X \subseteq Y$ iff $\Rightarrow_{X} \subseteq \Rightarrow_{Y}$.

Corollary 1. The mapping $\Rightarrow_{-}$is one-one on strongly minimal sets.

\section{$3 \quad$ Extracting constants from consequence relations}

\subsection{Defining extraction}

We now introduce an operation corresponding to the extraction of logical constants from a consequence relation. When a particular consequence relation is given, certain symbols are to be considered as logical constants because the consequence relation makes them play a special role with respect to validity. Our guiding intuition is that a symbol is constant if replacing it can destroy at least one inference. ${ }^{9}$

${ }^{8} u / u^{\prime}$ is the replacement which maps $u$ to $u^{\prime}$ but is the identity on all other symbols.

9 This is a variation on a similar idea first introduced in [4], Ch. 9. 
Definition 6. The function $C_{-}: C_{C N S_{L}} \rightarrow \wp\left(S y m b_{L}\right)$ is defined for $\Rightarrow \in$ $\mathrm{CONS}_{L}$ and $u \in$ Symb $_{L}$ by $u \in C_{\Rightarrow}$ iff there are $\Gamma, \varphi$ and $u^{\prime}$ such that $\Gamma \Rightarrow \varphi$ but $\Gamma\left[u / u^{\prime}\right] \nRightarrow \varphi\left[u / u^{\prime}\right]$.

Logical consequence can be construed as a function from sets of symbols to consequence relations. Extraction goes in the opposite direction. Moreover, the domains of both functions are naturally ordered by inclusion, so the situation is as shown in Figure 3.1. Proposition 2(b) said that $\Rightarrow_{-}$is an order-preserving mapping from $\left(\wp\left(S y m b_{L}\right), \subseteq\right)$ to $\left(C O N S_{L}, \subseteq\right)$. We would like $C_{-}$to provide some sort of inverse order-preserving mapping. Before looking into this and other properties of $C_{-}$, let us see some examples of how $C_{-}$works.

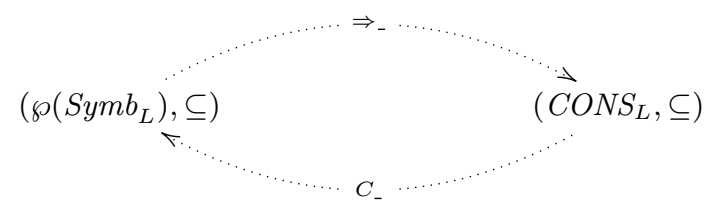

Fig. 1. Logical consequence and constant extraction

\subsection{Examples}

The function $C_{-}$might fail to yield the intended result because of its substitutional character. In particular, if a symbol $u$ is unique in its category, there is no other symbol to replace $u$ with, and trivially it will not count as a logical constant, no matter what inferential role it plays. This situation arises with negation, which is usually the only unary connective in the language. To sidestep this difficulty, let us assume, when considering propositional logic or first-order logic, that they come equipped with another unary connective, say $\dagger$, interpreted by the constant unary truth-function 'equal to false'. Then we get :

Proposition 6. $C_{\models_{P L}}$ is the standard set of logical constants of $P L$.

Let us see why in two examples. $p \models_{P L} p \vee q$ but $p \nvdash_{P L} p \wedge q$. Replacing $\vee$ by $\wedge$ destroys the validity of the first inference, so $\vee \in C_{\models_{P L}}$. Similarly, $\neg \neg p \models_{P L} p$ but $\dagger \dagger p \nvdash_{P L} p$, therefore $\neg \in C_{\models_{P L}}$. Similarly for quantifiers in first-order logic.

Proposition 7. $C_{\models_{F O}}=C_{\models_{F O} O_{s u b s t}}$ is the standard set of logical constants of first-order logic.

\subsection{Facts about $C_{-}$in the Bolzano setting}

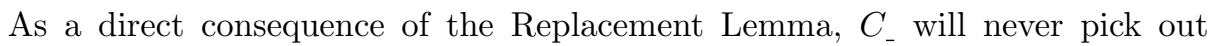
non-logical constants when it is applied to a Bolzano consequence. 
Proposition 8. For all $X \in \wp\left(\right.$ Symb $\left._{L}\right), C_{\Rightarrow_{X}} \subseteq X$.

Something stronger holds for strongly minimal $X$ :

Proposition 9. For all $X \in S_{M I N_{L}}, C_{\Rightarrow_{X}}=X$.

From this and Proposition 5, we get:

Proposition 10. $\Rightarrow_{-}$restricted to $S M I N_{L}$ is an isomorphism with inverse $C_{-}$.

This tells us that $C_{\text {_ }}$ plays its role as an order-preserving inverse mapping on some proper subset of $C O N S_{L}$, namely the Bolzano consequences generated from strongly minimal sets of constants. These are of course severe limitations to the scope of the result, and the remainder of this paper will be devoted to providing an understanding of the global picture. But in the present framework, $C_{-}$is simply not an order-preserving inverse on all of $C O N S_{L}$.

Proposition 11. There are languages $L$ and consequence relations $\Rightarrow$ and $\Rightarrow^{\prime}$ in $C O N S_{L}$ such that:

(a) $\Rightarrow \subseteq \Rightarrow^{\prime}$ but $C_{\Rightarrow} \nsubseteq C_{\Rightarrow}$

(b) $\Rightarrow \bar{\Phi} \Rightarrow_{C \Rightarrow}$

The failure of (a) in particular is no surprise given that there are both a positive and a negative condition in the definition of $C_{-}$. The witness to a non-valid inference might disappear by shifting to a bigger consequence relation. More surprisingly, the situation is no better for Bolzano consequences.

Proposition 12. There are languages $L$ and sets $X, Y \subseteq S y m b_{L}$ such that:

(a) $\Rightarrow_{X} \subseteq \Rightarrow_{Y}$ but $C_{\Rightarrow_{X}} \nsubseteq C_{\Rightarrow_{Y}}$

(b) $\Rightarrow_{X} \not \subset \Rightarrow_{C \Rightarrow_{X}}$

\section{Extra symbols}

The following example vividly illustrates the importance of having extra symbols available in a language. Let $S y m b_{L}=\{a, b\}, \operatorname{Sent}_{L}=\left\{R x y: x, y \in \operatorname{Symb}_{L}\right\}$, and $\operatorname{Tr}_{L}=\{R a a, R b b, R a b\}$. For example,

$$
\Rightarrow_{\emptyset} R a a,{ }^{10} \nRightarrow_{\emptyset} R a b, \quad \Rightarrow_{\{a\}} R a b
$$

Here, you must move two symbols in order to turn a true sentence into a false one. As a result, $C_{-}$picks no constants at all: for all $X \subseteq S y m b_{L}, C_{\Rightarrow_{X}}=\emptyset$. However, expand $L$ conservatively to $L^{\prime}$ by adding at least one new symbol $c$, while $\operatorname{Tr}_{L}=\operatorname{Tr}_{L^{\prime}} \cap S_{e n t_{L}}$. Then, regardless of the truth values of new sentences in $L^{\prime}$ :

(6) In $L^{\prime}$, for all $X \subseteq \operatorname{Symb}_{L}, C_{\Rightarrow_{X}}=X$.

For example, we now have $\Rightarrow_{\{a\}} R a b$ in $L^{\prime}$ (by conservativity), but $\nRightarrow_{\{a\}} R c b$, (by the replacement $\rho(c)=b, \rho(b)=a)$. Thus, in $L^{\prime}, a \in C_{\Rightarrow_{\{a\}}}$.

$\overline{{ }^{10} \text { Writing } \Rightarrow_{X}} \varphi$ for $\emptyset \Rightarrow_{X} \varphi$, i.e. $\varphi$ is valid relative to the constants in $X$. 


\subsection{Richness and abundance}

One way of making extra symbols available is to simply assume that there are infinitely many symbols of each category in $L$. Call such languages rich. Note that nothing prevents most of these symbols from meaning the same.

Proposition 13. If $L$ is rich, then $\Rightarrow_{X} \subseteq \Rightarrow_{Y}$ implies $\Rightarrow_{X}=\Rightarrow_{X \cap Y}$. In particular, for any $X$, the set $\left\{Z: \Rightarrow_{Z}=\Rightarrow_{X}\right\}$ is closed under finite intersections, so if $X$ is finite, this set has a smallest element.

Proposition 14. If $L$ is rich, and $X$ is minimal and finite, then $C_{\Rightarrow_{X}}=X$.

Using a variant of the earlier example, one can show that the assumption of finiteness in this proposition is essential.

Corollary 2. If $L$ is rich, then for finite $X, \Rightarrow_{X}=\Rightarrow_{C \Rightarrow_{X}}$.

Corollary 3. If $L$ is rich, then every finite $X \subseteq S y m b_{L}$ has a unique smallest subset, namely $C_{\Rightarrow_{X}}$, that generates $\Rightarrow_{X}$.

Here is an even stronger requirement on $L . u$ and $u^{\prime}$ (of the same category) are synonymous,

$$
u \equiv_{L} u^{\prime}
$$

iff replacing (some) occurrences of $u$ by $u^{\prime}$ or vice versa does not change the truth value of $L$-sentences. We say that $L$ is abundant iff there are infinitely many synonyms of each symbol. So all the results above hold for abundant $L$, but in addition we have

Proposition 15. If $L$ is abundant, minimality and strong minimality coincide.

Another variant of our example shows that this can fail when only richness is assumed.

Corollary 4. If $L$ is abundant, the results of Proposition 14 and Corollaries 2 and 3 hold for infinite $X$ as well.

\subsection{Expansions}

Richness and abundance may seem a bit extravagant assumptions. What one really needs, however, is the ability to add new symbols to $L$, in particular symbols with the same meaning as old ones, i.e. synonyms or copies. We now slightly revise our Bolzano set-up to make this possible.

Recall that an interpreted language $L$, as we defined it, comes with sets $\operatorname{Symb}_{L}$, Sent $_{L}$, and $\operatorname{Tr}_{L}$; in fact, we may set $L=\left\langle S y m b_{L}, \operatorname{Sent}_{L}, \operatorname{Tr}_{L}\right\rangle$. We say that $L^{\prime}$ is an expansion of $L, L \leq L^{\prime}$, iff $\operatorname{Symb}_{L} \subseteq \operatorname{Symb}_{L^{\prime}}$, Sent $_{L}=\left\{\varphi \in\right.$ Sent $_{L^{\prime}}$ : $\left.V_{\varphi} \subseteq S y m b_{L}\right\}$, and $\operatorname{Tr}_{L}=\operatorname{Tr}_{L^{\prime}} \cap S_{\text {Sent }} . L^{\prime}$ is an expansion with copies, $L \leq_{c} L^{\prime}$, iff in addition every new symbol is synonymous, in $L^{\prime}$, with some $L$-symbol. 
Proposition 16. $\leq$ is a partial order (reflexive, antisymmetric, and transitive), and $\leq_{c}$ is a sub-order.

A partially ordered set $Z$ is directed iff it is upward closed: if $a, b \in Z$ there is $c \in Z$ such that $a \leq c$ and $b \leq c$. Now, our idea is to replace the fixed language $L$ with a directed family $\mathcal{L}$ of expansions of $L$. This requires a slight reformulation of what we have done so far. In what follows, $\mathcal{L}$ is any directed family of expansions of $L$. To start with Bolzano consequence, suppose $\Gamma \cup\{\varphi\} \subseteq$ Sent $_{L}$ and $X \subseteq$ Symb $_{L}$.

Definition 7. $\Gamma \Rightarrow_{X, L} \varphi$ iff for every $L^{\prime} \in \mathcal{L}$ and every replacement $\rho$ in $L^{\prime}$ (for $\Gamma$ and $\varphi$ ) which is the identity on $X$, if $\Gamma[\rho] \subseteq \operatorname{Tr}_{L^{\prime}}$, then $\varphi[\rho] \in \operatorname{Tr}_{L^{\prime}}$.

The family $\mathcal{L}$ is suppressed in this notation, and has to be made clear in context. If $\mathcal{L}=\{L\}$, we have our previous notion of Bolzano consequence: $\Rightarrow_{X, L}=\Rightarrow_{X}$. Normally, the sentences we talk about will belong to several languages in $\mathcal{L}$.. That this is not a problem follows from

Lemma 3. If $\Gamma \cup\{\varphi\} \subseteq$ Sent $_{L}, X \subseteq$ Symb $_{L}$, and $L^{\prime} \in \mathcal{L}$, then

$$
\Gamma \Rightarrow_{X, L} \varphi \text { iff } \Gamma \Rightarrow_{X, L^{\prime}} \varphi
$$

where the right-hand side is relative to the subclass $\mathcal{L}^{\prime}=\left\{L^{\prime \prime} \in \mathcal{L}: L^{\prime} \leq L^{\prime \prime}\right\}$.

In what follows, when $\mathcal{L}$ is given and $L^{\prime} \in \mathcal{L}$, we always understand $\Rightarrow_{X, L^{\prime}}$ to be relative to the corresponding subfamily generated by $L^{\prime}$.

Next, we extend the notion of (strong) minimality to the new set-up: $X$ is minimal iff for each $u \in X$ there is $L^{\prime} \in \mathcal{L}$ such that $\Rightarrow_{X, L^{\prime}} \neq \Rightarrow_{X-\{u\}, L^{\prime}}$, and analogously for strong minimality. Finally, we extend the definition of $C_{-}$to the case of consequence relations of the form $\Rightarrow_{X, L}$. Let $u \in S y m b_{L}$.

Definition 8. $u \in C_{\rightrightarrows_{X, L}}$ iff there are $L^{\prime} \in \mathcal{L}, \Gamma \cup\{\varphi\} \subseteq$ Sent $_{L^{\prime}}$, and $u^{\prime} \in$ Symb $_{L^{\prime}}$ such that $\Gamma \Rightarrow_{X, L^{\prime}} \varphi$ but $\Gamma\left[u / u^{\prime}\right] \nexists_{X, L^{\prime}} \varphi\left[u / u^{\prime}\right]$.

Call a family $\mathcal{L}$ is copy-closed iff for every $L^{\prime} \in \mathcal{L}$ and every $A \subseteq S y m b_{L^{\prime}}$ there are $L^{\prime \prime} \in \mathcal{L}$ and $B \subseteq S y m b_{L^{\prime \prime}}$ such that $B \cap S_{y m b} b_{L^{\prime}}=\emptyset$ and there is a copy $b \in B$ of every $a \in A$. A simple case of a copy-closed family is $\operatorname{copies}(L)=$ $\left\{L^{\prime}: L \leq_{c} L^{\prime}\right\}$. This is essentially the interpreted language $L$ with the possibility of adding (arbitrarily many) new names of already named things. Thus, it is a very mild extension of the original Bolzano set-up. Now all results assuming richness or abundance from the previous subsection hold automatically relative to copy-closed families, without any further assumptions:

Proposition 17. Relative to a copy-closed directed family of expansions of $L$ :

(a) Minimality and strong minimality coincide.

(b) For all $X \subseteq$ Symb $_{L}, \Rightarrow_{X, L}=\Rightarrow_{C \Rightarrow_{X, L}}$.

(c) Each $X \subseteq S y m b_{L}$ has a unique smallest subset, $C_{\rightrightarrows_{X, L}}$, generating $\Rightarrow_{X, L}$. 


\section{Galois connections}

\subsection{General consequence relations}

We generalized our initial framework by working with directed families of expansions of $L$. In terms of Figure 3.1, the sets of symbols we are interested in are still subsets $S y m b_{L}$. But such sets generate consequence relations for $L$ as well as for the expansions of $L$. In particular, the extended Definition 8 of $C_{\text {- appeals }}$ not only to the consequence relation of $L$ but also to those of the expansions. As a result, we need to extend our notion of a consequence relation.

Definition 9. A general consequence relation for a family of languages $\mathcal{L}$ is a family of consequence relations $\Rightarrow=\left\{\Rightarrow_{L^{\prime}}\right\}_{L^{\prime} \in \mathcal{L}}$ such that for all $L^{\prime}, L^{\prime \prime} \in \mathcal{L}$ with $L^{\prime} \leq L^{\prime \prime}, \Rightarrow_{L^{\prime}} \subseteq \Rightarrow_{L^{\prime \prime}}$.

For $X \subseteq \operatorname{Symb}_{L}$, let $\Rightarrow_{X}$ be the family $\left\{\Rightarrow_{X, L^{\prime}}\right\}_{L^{\prime} \in \mathcal{L} .} \Rightarrow_{X}$ is a general consequence relation. Note that the extended Definition 8 of $C_{\text {- }}$ applies not just to $\Rightarrow_{X, L}$ but to any general consequence relation. Given a directed family $\mathcal{L}$ of expansions of $L$, we let $G C O N S_{L}$ be the class of general consequence relations for $\mathcal{L}$ and $B G C O N S_{L}$ the class of general consequence relations of the form $\Rightarrow_{X}$.

\subsection{Galois connection for copies}

What kind of correspondence do we get between $\Rightarrow_{X}$ and $C_{-}$in this setting? We want something as close as possible to an isomorphism, with as few assumptions as possible on $\mathcal{L}$. A relevant notion of correspondence in that context is the notion of Galois connection. A Galois connection between two ordered sets $A$ and $B$ is a pair $(f, g)$ of functions, with $f: A \rightarrow B$ and $g: B \rightarrow A$, such that the following four conditions hold: (1) $f$ is monotone, (2) $g \circ f$ is decreasing, (3) $g$ is monotone, (4) $f \circ g$ is increasing. $f$ is then an isomorphism with inverse $g$ from $g(B)$ to $f(A)$. Intuitively, $f$ and $g$, even though they do not constitute a full-blown isomorphism, give rise to one between the sufficiently well-behaved subsets $g(B)$ and $f(A)$.

Proposition 18. Relative to a copy-closed family, the following hold:

(a) $\Rightarrow_{X} \subseteq \Rightarrow_{Y}$ implies $C_{X} \subseteq C_{\rightrightarrows_{Y}}$

(b) $\Rightarrow_{X} \subseteq \Rightarrow_{C_{X}}$

Considering $\left(\Rightarrow_{-}, C_{-}\right)$as a tentative Galois connection between $\wp\left(S y m b_{L}\right)$ and $G C O N S_{L}$, conditions (1) and (2) are always satisfied. (a) and (b) in Proposition 18 are (3) and (4), when the class of general consequence relations is restricted to $B G C O N S_{L}$. We have shown:

Theorem 1. $\left(\Rightarrow_{-}, C_{-}\right)$is a Galois connection between $\wp\left(S y m b_{L}\right)$ and $B G C O N S_{L}$ for copy-closed families of languages. 
The set $C_{B G C O N S_{L}}$ of sets of symbols which are the image of some $\Rightarrow_{X}$ in $B G C O N S_{L}$ under $C_{-}$is of special interest, since it is the restriction of $\wp\left(S y m b_{L}\right)$ for which $\Rightarrow_{-}$is an isomorphism.

Proposition 19. Relative to a copy-closed family of expansions of $L, C_{B G C O N S_{L}}$ is the set of minimal sets in $\wp\left(S_{y m b}\right)$.

On the left-hand side of our picture, the well-behaved objects in $\wp\left(S_{y m b}\right)$ are the minimal sets. On the right-hand side, our Galois connection has the nontypical property that $\Rightarrow_{\text {_ }}$ is onto. If we were to extend the picture to include not only $B G C O N S_{L}$ but all of $G C O N S_{L}$, we would get that the part of $G C O N S_{L}$ for which we have the isomorphism is precisely $B G C O N S_{L}$, but the properties stated in Proposition 18 for relations in $B G C O N S_{L}$ do not hold for all relations in $G C O N S_{L}$.

Question: Is there a natural class $G C O N S_{L}^{*} \supseteq B G C O N S_{L}$ for which they hold?

\subsection{Tarskian consequence}

copies $(L)$ is the simplest case of a copy-closed family of languages. A maximal case is the family of all expansions of a base-language $L$. Instead of just fixing a set of true sentences, consider interpreted languages $L$ for which truth is defined as truth with respect to a fixed intended interpretation $I$ of symbols of $L$. Let ' $J \vDash \varphi$ ' abbreviate ' $\varphi$ is true according to interpretation $J$ '. Tarskian consequence (with a fixed domain of interpretation) can then be defined:

Definition 10. $\Gamma \vDash_{X} \varphi$ iff for all $J$ such that $J \approx_{X} I$, if $J \vDash \Gamma$, then $J \vDash \varphi$.

$\left(J \approx_{X} I\right.$ means that $J$ and $I$ agree on $X \subseteq S y m b_{L}$.) Tarskian consequence is equivalent to Bolzano consequence with respect to the family of all expansions:

Proposition 20. $\Gamma \vDash_{X} \varphi$ iff for every $L^{\prime} \geq L$ and every replacement $\rho$ that keeps all elements in $X$ fixed, if $I^{\prime} \vDash \Gamma[\rho]$, then $I^{\prime} \vDash \varphi[\rho]$.

(Here $I^{\prime}$ is an interpretation extending $I$ to the new symbols in $S y m b_{L^{\prime}}$.) Together with Proposition 18 and the fact that the family of all expansions is copy-closed, this implies that there is a Galois connection between $\wp\left(S y m b_{L}\right)$ and the class of Tarskian consequence relations.

\section{References}

1. P. Aczel, Replacement systems and the axiomatization of situation theory, in R. Cooper, K. Mukai, and J. Perry (eds.), Situation Theory and its Applications, Vol. 1, CSLI Publications, Stanford, 1990, 3-33.

2. J. van Benthem, Is there still logic in Bolzano's key?, in E. Morscher (ed.), Bernard Bolzanos Leistungen in Logik, Mathematik und Physik Bd., 16, Academia Verlag, Sankt Augustin, 2003, 11-34.

3. M. Dunn and N. Belnap Jr., The substitution interpretation of the quantifiers, Noûs 4 (1968), 177-185.

4. S. Peters and D. Westerståhl, Quantifiers in Language and Logic, Oxford UP, Oxford, 2006. 